\title{
Promising Anticancer Potential of Herbal Compounds against Breast Cancer: A Systemic Review
}

Mrudul Vekaria* and Pravin Tirgar

\author{
School of Pharmacy, R.K. University, Rajkot-360020, Gujarat, India; vekaria_mrudul@yahoo.com
}

\begin{abstract}
Breast cancer (BC) exhibit a high level of mortality rate and incidence but a comprehensive treatment is unavailable, which can successfully eradicate this fatal disease. Novel therapeutic strategies have been intensively studied to eradicate $\mathrm{BC}$, including the use of traditional medicines and phytochemical (herbal compounds). These compounds have been studied in many aspects, such as targeting cell cycle and key tumor-associated metabolic pathways. In this study, we reviewed the literature for the potential therapeutic application of herbal compounds against breast cancer. Several terms, including "Herbal compounds", "Breast Cancer" and "Traditional medicines", were searched in PubMed and Web of Science databases. The search results were narrowed down using many filters and the resultant literature was critically analyzed. The enormous number of articles showed the promising potential of herbal compounds as anticancer therapy. The comprehensive evaluation of the literature suggested the anti-angiogenic, anti-proliferative, anticancer stem cells, anti-inflammatory/antioxidant, anti-metastatic, and epigenetic effects of herbal compounds as anticancer effects against BC. Despite this enormous data, there is lack of sufficient demonstration of the consumption of herbal compounds in a regular as anticancer strategy in clinical trials. Therefore, given its promising anticancer potential, focus should be shifted towards the regular use of herbal compounds in anticancer strategies.
\end{abstract}

Keywords: Anticancer Strategy, Breast Cancer, Herbal Compounds, Traditional Medicines

\section{Introduction}

Cancer is known to be the major cause of mortality worldwide along with cardiovascular diseases, despite of advancement in research and diagnosis. In the whole world, breast cancer is considered to be the second most occurring neoplasia among women ${ }^{1}$. According to an estimate, nearly 500,000 deaths of the total 2 million breast cancer patients are recorded per year. This rapidly increasing number of breast cancer patients in the $21^{\text {st }}$ century implies the epidemic status of this disease $\mathrm{e}^{1}$. Cancer chemoprevention is considered to be an effective tool to minimize the threat of cancer development. This chemoprevention is known to target both the epigenetic changes that are responsible for sporadic breast cancer and genetic factors of breast carcinogenesis. Epigenetic modifications leading to sporadic breast cancer is responsible for $90 \%$ of all cases ${ }^{1}$. Moreover, the chemoprevention is regarded as effective, less toxic and cost effective in comparison to the therapy done to treat breast cancer.

Phytochemicals are abundantly present in the eatable parts of plant which reduces the occurrence of human disease particularly cancer ${ }^{2,3}$. Epidemiological, preclinical, and clinical trials proposes that plants containing phytochemicals, such as phenolic compounds, terpenoids and flavonoids etc. exhibit anticancer and other significant biological effects, including anti-allergic, anti-

${ }^{*}$ Author for correspondence 
aggregagoty, anti-inflammatory and neuro-protective, effects ${ }^{4}$. The phytochemicals are known to target the varied molecular pathways in cancer development such as angiogenesis, cancer stem cells, proliferation, inflammation, cell infiltration, apoptosis, or epigenome, without causing detrimental effects in cells. Research in this area firmly proposes that the synergistic or additive effects of phytochemicals found in the foods derived from whole plant might be linked with their substantial anticancer and pleiotropic properties ${ }^{5}$. It is assumed that the single molecule derived from plants does not cause the same biological activity as the molecules derived from whole plant. It means that the effect of a single molecule is much weaker as compared to the synergistic effects produced by the molecules derived from whole plant ${ }^{2,6}$. Alternative medical care systems e.g., Chinese, Ayurvedic belonging to traditional medicine have been widely used over thousands of years as a potent source in suppressing cancer growth. The plants, spices and herbs derived molecules and their combined formulas are used in traditional medicine and could contribute as an anticancer agent and chemoprevention in carcinogenesis without producing harmful effects. Thus, the use of traditional medicine owing to their boundless benefits has gained prime importance in the area of cancer research ${ }^{6,7}$. The combined effects of numerous phytochemicals derived from various plant substances in traditional medicine poses significant tumor suppressive effects. They target the cell signaling pathways that considered being effective in reduction of carcinogenesis in high-risk individuals. Consequently, the use of traditional medicine should be considered an effective therapeutic approach in oncology research.

In this study, we searched different databases for mining the literature related to potential anticancer effects of herbal compounds and their mechanism of actions were critically studied and reviewed.

\section{Methodology}

A workflow of the methodology adopted is presented in Figure 1.

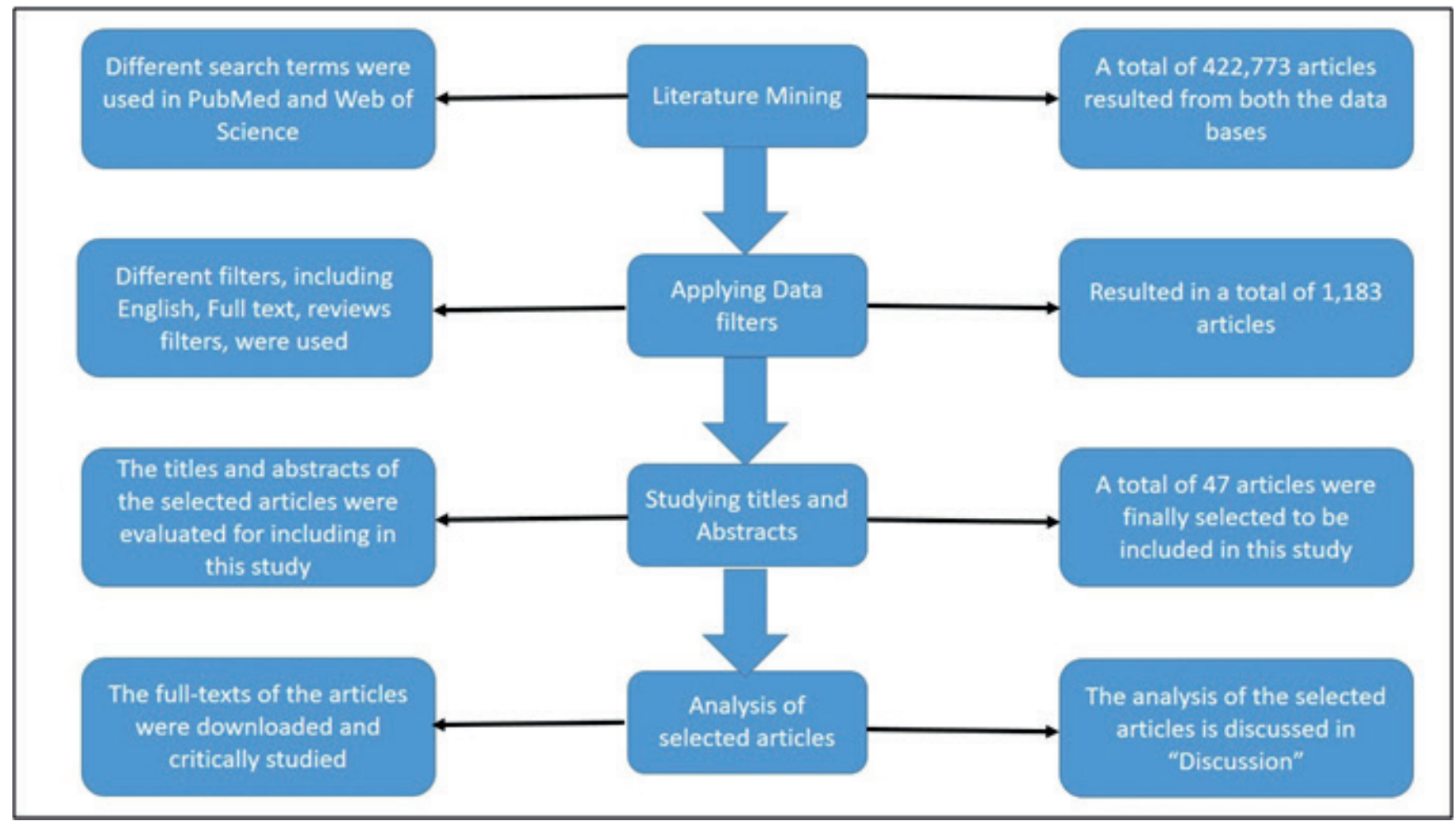

Figure 1. Workflow of the study.

\subsection{Data Mining}

The articles were searched and retrieved from PubMed and Web of Science databases. Different search terms were used, which included "Cancer", "Herbal compounds" and
"Anticancer effects of herbal compounds". The resulted articles in both of the databases were in a very huge number. Therefore, many filters were used to narrow down the search results. First, the search results were 
filtered for full-text articles, followed by filtering for English language. The resulted data was still huge, which was then filtered to skip the review articles, including narrative reviews, systemic reviews and meta-analysis. Then, the search results were filtered for "Breast Cancer" and "Traditional medicines", which narrowed down the search results to a number which could be studied.

\subsection{Data Assessment}

The assessment of the search results were carried out in two steps. In the first step, the titles of the articles were studied and those did not match the requirements of this study were excluded. In the second step, the abstracts of the selected were studied and if the article fulfilled our requirements were selected. The full texts of the selected articles were downloaded and critically analyzed.

\section{Results}

\subsection{Data Assessment}

From the initial data search, the results from both the databases included 422,773 articles. To study this huge number of articles was out of our capacity. Therefore, the filters, including full-text, English and reviews filters, were applied, which narrowed down the results to 344,983 articles, 334, 213 articles and 290,271 articles, respectively. The addition of more filters to narrow down data to those related to breast cancer only surprisingly lowered the number of articles to a total of 1,183 . The year-wise distribution of all articles from both the databases is presented in Figure 2.

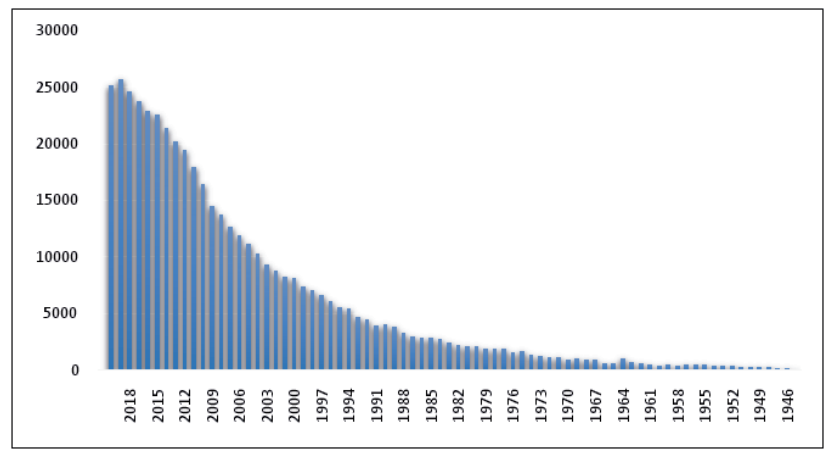

Figure 2. Year-wise distribution of all the articles from PubMed and Web of Science.

\subsection{Assessment of the Data}

The titles of all the selected 1,183 were studied. It was found that some of the articles did not fit our requirement, which were, therefore, skipped. The abstracts of the selected articles were studied and their full texts were downloaded. Finally, a total of 47 articles were included in the study (Table 1-3).

\subsection{Analysis of the Potential Anticancer Herbal Compounds}

The full-text articles were critically studied and the articles were divided into two categories. The first categories included the anticancer effects of the herbal compounds against all cancer types (Table 1-3). The second category included articles that focused on the potential anticancer effects of herbal compounds against breast cancer. The articles from the second category were then critically analyzed.

\section{Discussion}

\subsection{Targets of Herbal Medicinal Compounds}

\subsubsection{Targeting the Cell Cycle}

The phytochemical given to the cancer cell lines cause changes in cell cycle. Phytochemicals alters the cell signaling pathways and modulates the apoptosis, proli feration and survival of cancer cells $\mathrm{s}^{51,52}$. The interaction of Cyclin-Dependent Kinase Inhibitors (CDKIs) and cyclin which are the two subunits of Cyclin-Dependent Kinases (CDKs) is necessary for its activity. The CDKs can be regulated to arrest the cell cycle at any stage ${ }^{53}$. The reduced expression of Cyclin D1 and E, down regulation of CDK4/6 and CDK2, by various phytochemicals in particular flavonoids or curcumin is due to their ability to arrest the cell cycle at g1 phase in cancer cell ${ }^{54,55}$. Various phytochemicals causes increase in the synthesis of CDK1 and it is binding with CDK which inhibits the activity of CDK, known to be responsible for the transition from G1 to $S$ phase ${ }^{56}$. Numerous phytonutrients exhibited a strong inhibition and arrested the cell cycle at G2 phase ${ }^{57,58}$. During the G2-M transition-linked cellular changes, a fundamental role was demonstrated by $\mathrm{Cdc} 2$ kinase activity and the modulations in chk1/2, cdc25 and cyclin B1, along with p21/cip1 and p53, were effected significantly. The phytochemicals of soy and garlic such as curcumin and isoflavones have been reported to reduce the activity of CDC2 kinase and arrest the cell cycle at G2-M checkpoint, resulting in the inhibition of the proliferation of tumor cell ${ }^{58-60}$. 


\begin{tabular}{|c|c|c|c|c|c|c|c|c|c|c|c|}
\hline$\stackrel{\dot{\Xi}}{\mathscr{\Xi}}$ & $\infty$ & $a$ & 으 & $\exists$ & $\stackrel{\circ}{-}$ & $\beth$ & $\simeq$ & $\stackrel{m}{-}$ & $\underset{\Xi}{\ddagger}$ & $\stackrel{20}{n}$ & $\underline{-1}$ \\
\hline 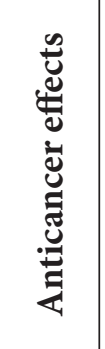 & 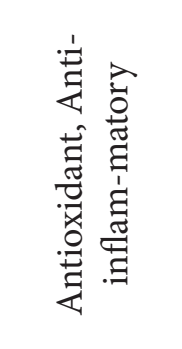 & 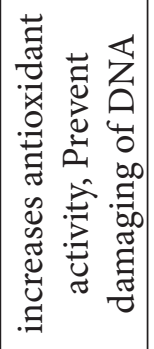 & 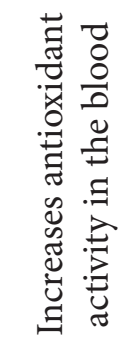 & 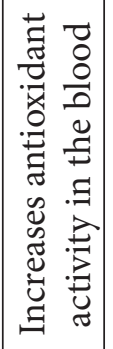 & 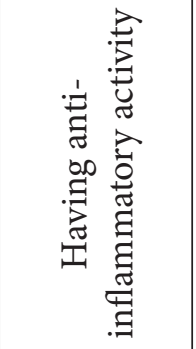 & & & 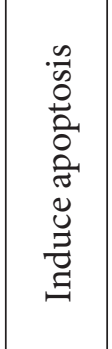 & 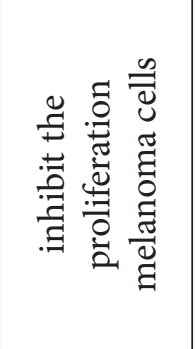 & 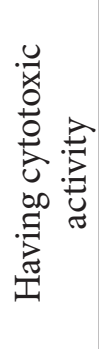 & 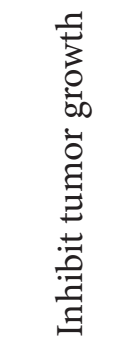 \\
\hline 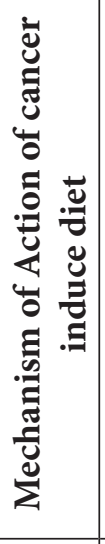 & 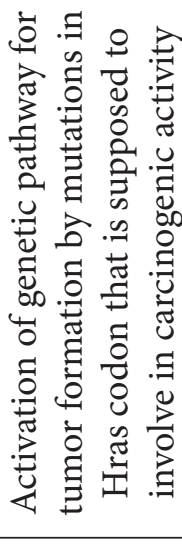 & 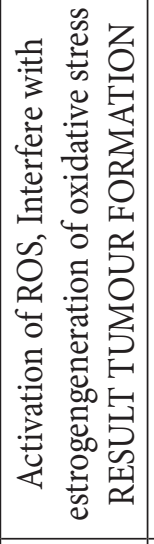 & 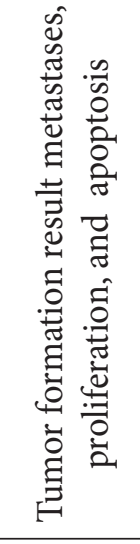 & 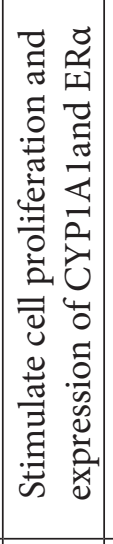 & 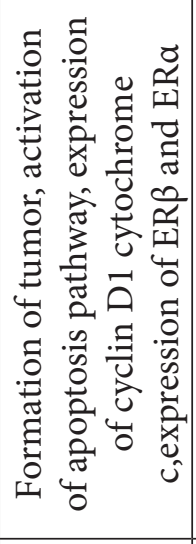 & 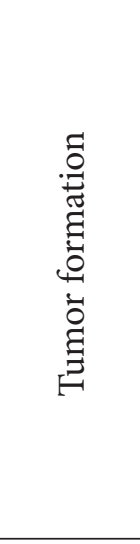 & 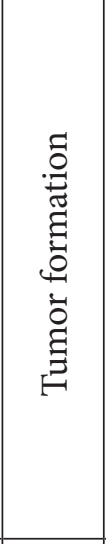 & 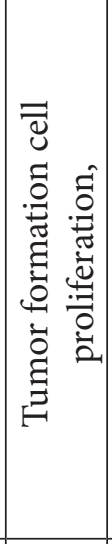 & 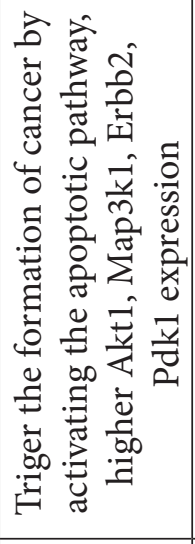 & 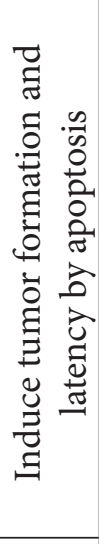 & 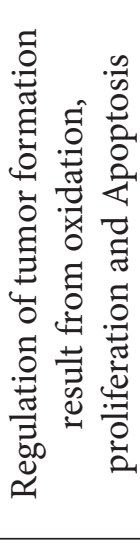 \\
\hline 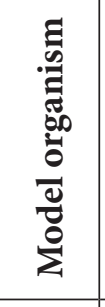 & 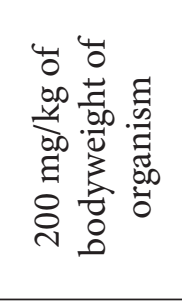 & 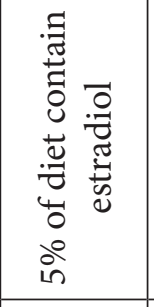 & 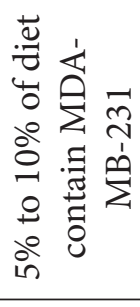 & 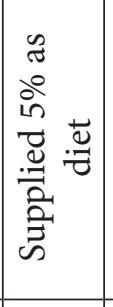 & 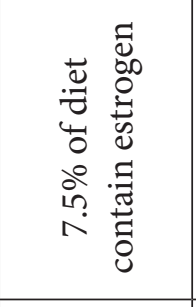 & 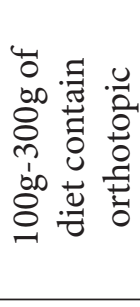 & 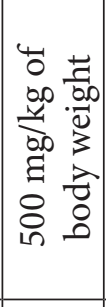 & 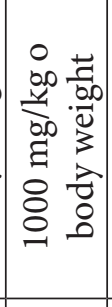 & 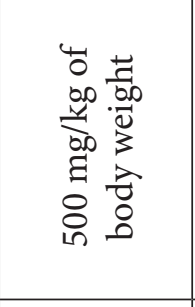 & 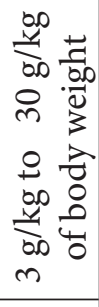 & 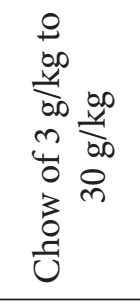 \\
\hline 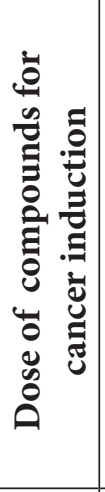 & 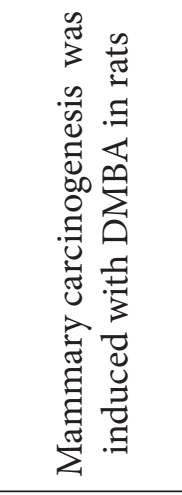 & 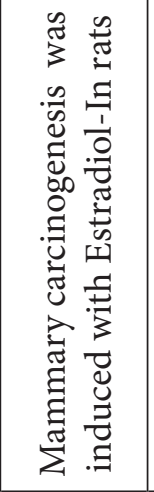 & 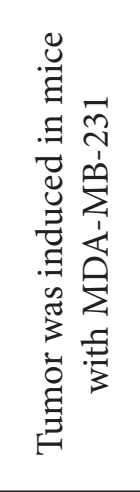 & 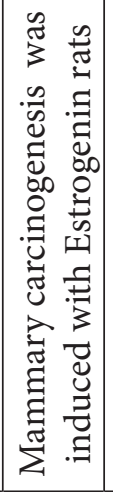 & 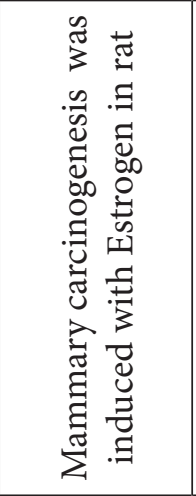 & 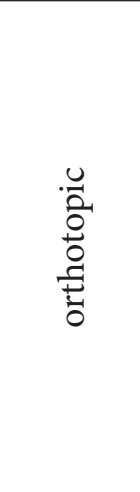 & 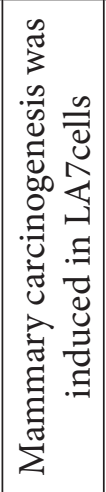 & 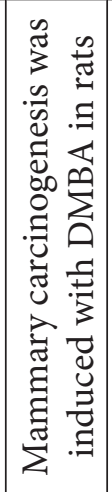 & 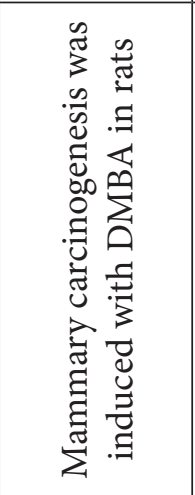 & 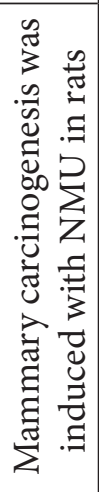 & 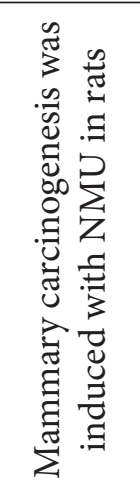 \\
\hline 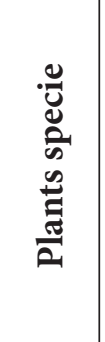 & 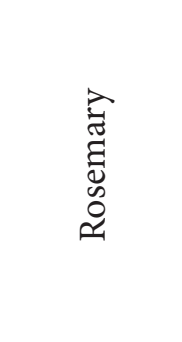 & 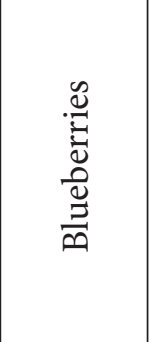 & 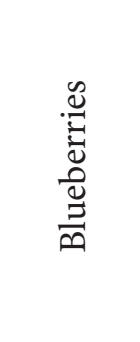 & 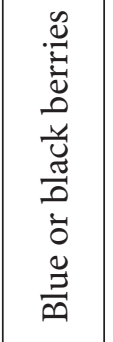 & 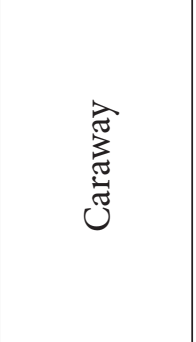 & 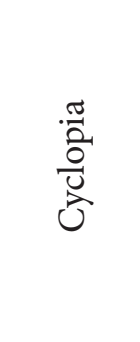 & 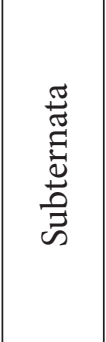 & 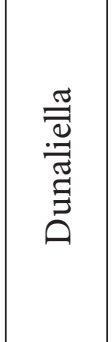 & 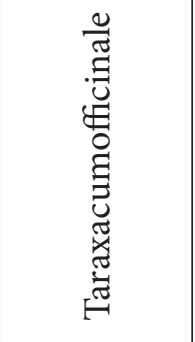 & 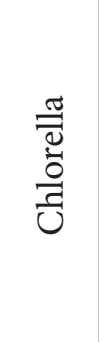 & 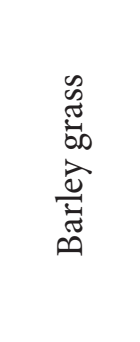 \\
\hline
\end{tabular}




\begin{tabular}{|c|c|c|c|}
\hline$\beth$ & $\stackrel{\infty}{-}$ & $\curvearrowright$ & $\sqrt{7}$ \\
\hline 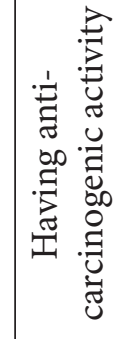 & 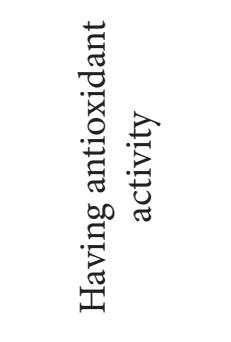 & 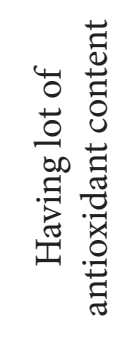 & \\
\hline 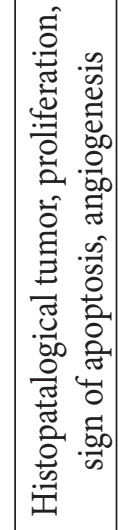 & 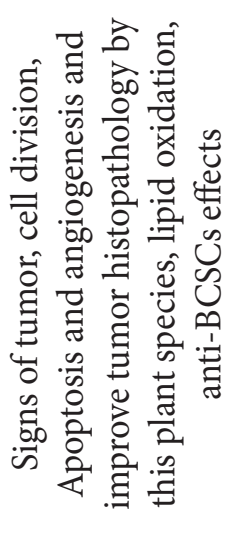 & 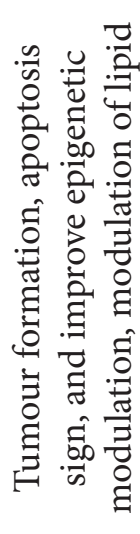 & 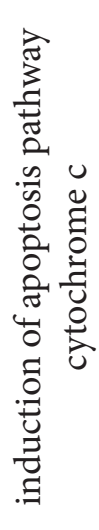 \\
\hline 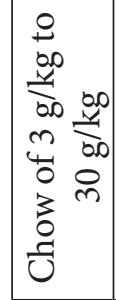 & 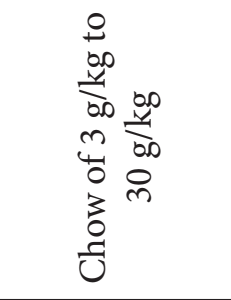 & 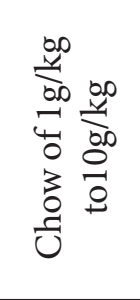 & 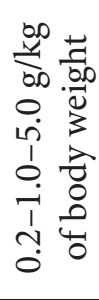 \\
\hline 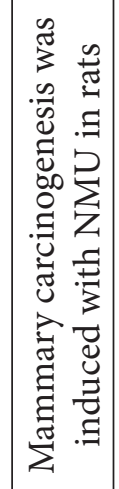 & 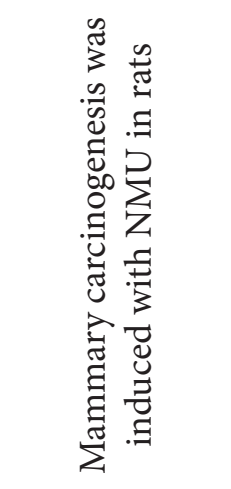 & 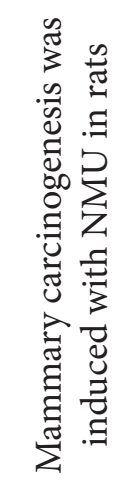 & 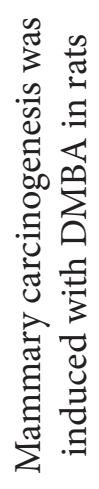 \\
\hline 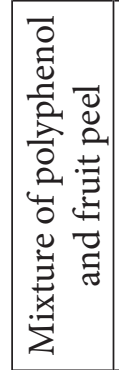 & 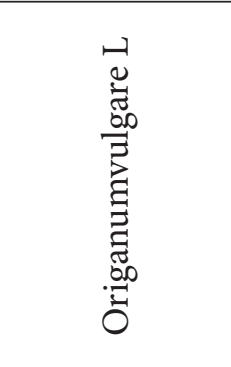 & 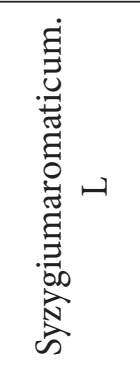 & 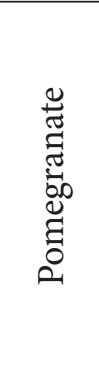 \\
\hline
\end{tabular}

Table 3. Studies reporting the inhibition of pathways in breast cancer studies by plants-derived compounds

\begin{tabular}{|c|c|c|c|}
\hline $\begin{array}{c}\text { Natural } \\
\text { compound }\end{array}$ & $\begin{array}{l}\text { Chemothera- } \\
\text { peutic drug }\end{array}$ & $\begin{array}{c}\text { Signal } \\
\text { pathway }\end{array}$ & Refs. \\
\hline Curcumin & Rhtrail & $\begin{array}{c}\text { FEN1 } \\
\text { DR5/IAP }\end{array}$ & 36,37 \\
\hline Quercetin & Rhtrail & $\begin{array}{c}\text { c-FLIP/ } \\
\text { DR5 }\end{array}$ & 38 \\
\hline Emodin & Tamoxifen & Ras/ERK & 39 \\
\hline Resveratrol & Doxorubicin & HSP-27 & 40 \\
\hline Resveratrol & Doxorubicin & $\begin{array}{c}\text { Carbonyl } \\
\text { reductase } 1\end{array}$ & 41 \\
\hline Formononetin & Metformin & $\begin{array}{c}\text { ERK1/2/ } \\
\text { Bcl-2 }\end{array}$ & 7 \\
\hline Crassin & Doxorubicin & $\begin{array}{l}\text { Apopto- } \\
\text { sis-ROS }\end{array}$ & 42 \\
\hline Enterolactone & + & Apoptosis & 43 \\
\hline $\begin{array}{l}\text { Secoisolari- } \\
\text { ciresinol }\end{array}$ & + & $\begin{array}{c}\text { Antipro- } \\
\text { liferative, } \\
\text { pathway } \\
\text { ROS pro- } \\
\text { duction } \\
\text { pathway. }\end{array}$ & 43 \\
\hline $\begin{array}{l}\text { Nordihydro- } \\
\text { guaiareticacid }\end{array}$ & Cisplatin & ROS & 44 \\
\hline $\begin{array}{c}\text { Trans-nero- } \\
\text { lidol }\end{array}$ & Doxorubicin & $\begin{array}{l}\text { Doxorubi- } \\
\text { cin accu- } \\
\text { mulation }\end{array}$ & 45 \\
\hline Wogonin & Doxorubicin & Nrf2 & 46 \\
\hline Biochanin A & Daunorubicin & $\begin{array}{l}\text { Not investi- } \\
\text { gated }\end{array}$ & 47 \\
\hline Glabridin & Doxorubicin & $\begin{array}{c}\text { Not investi- } \\
\text { gated }\end{array}$ & 48 \\
\hline Ursolic acid & Doxorubicin & $\begin{array}{c}\text { Not investi- } \\
\text { gated }\end{array}$ & 49 \\
\hline Mangiferin & + & $\begin{array}{l}\text { p-Glyco- } \\
\text { protein, }\end{array}$ & 50 \\
\hline
\end{tabular}




\begin{tabular}{|c|c|c|c|c|c|c|c|c|c|c|c|c|c|c|c|c|}
\hline$\stackrel{\oplus}{\mathscr{U}}$ & ㄱ & $\vec{\sim}$ & $\widetilde{N}$ & $\ddot{\sim}$ & $\stackrel{\sim}{\sim}$ & $\stackrel{\stackrel{2}{\sim}}{ }$ & $\stackrel{\circ}{\sim}$ & $\widehat{ત}$ & $\stackrel{\infty}{\sim}$ & ๙ิ & ిల & $\vec{m}$ & m & $m$ & mু & $\stackrel{n}{m}$ \\
\hline 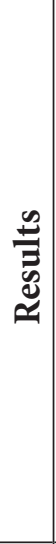 & 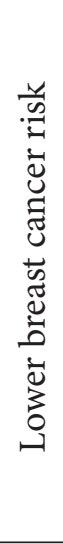 & 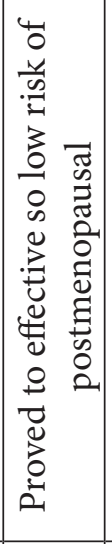 & 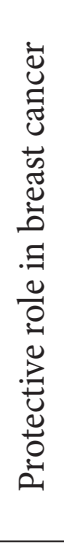 & 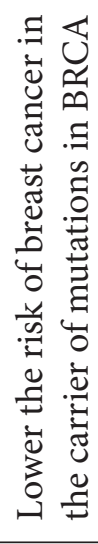 & 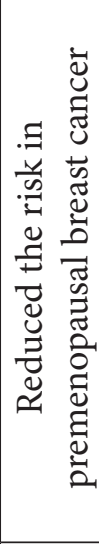 & 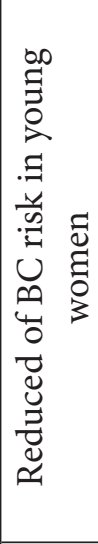 & 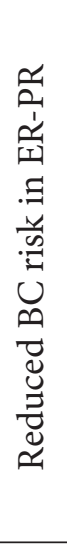 & 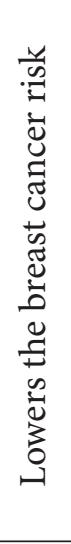 & 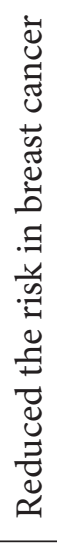 & 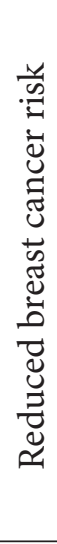 & 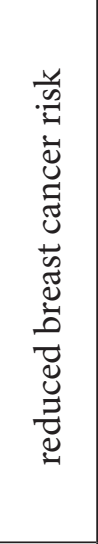 & 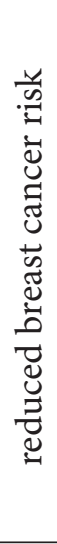 & 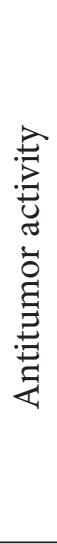 & 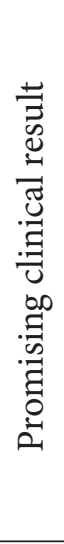 & 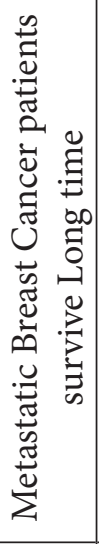 & 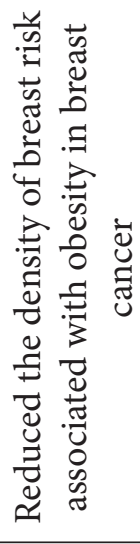 \\
\hline 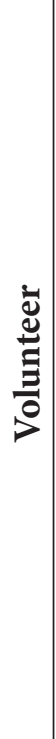 & 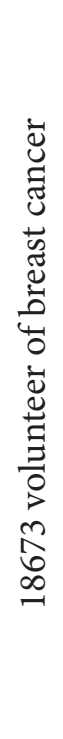 & 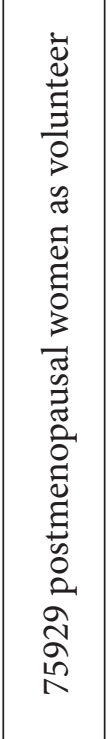 & 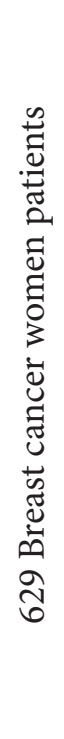 & 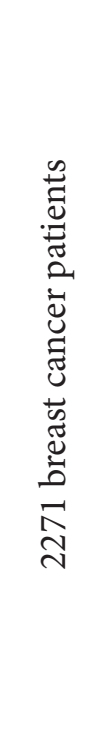 & 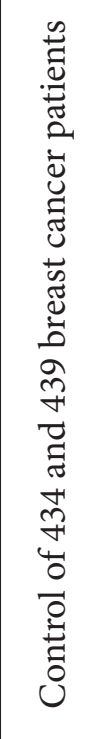 & 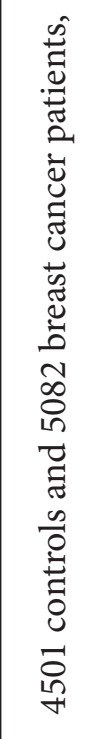 & 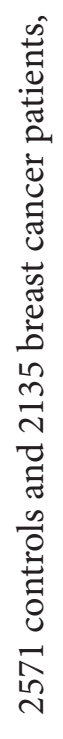 & 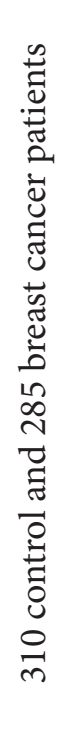 & 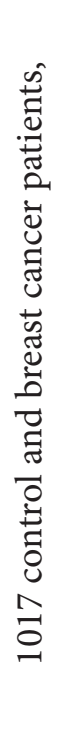 & 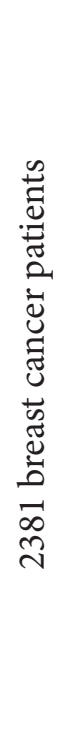 & 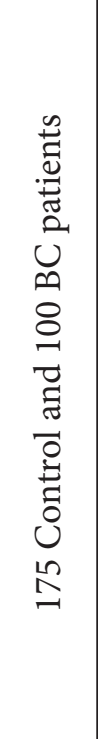 & 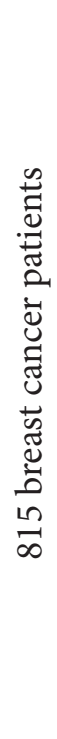 & 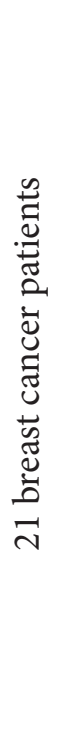 & 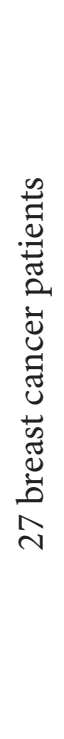 & 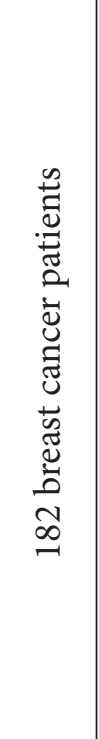 & 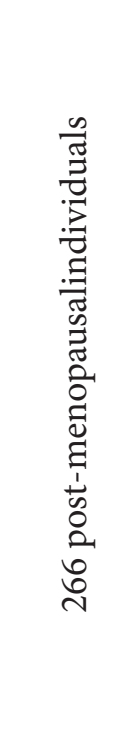 \\
\hline 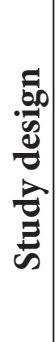 & 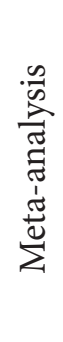 & 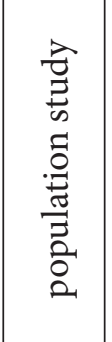 & 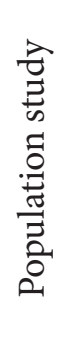 & 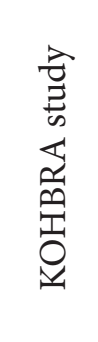 & 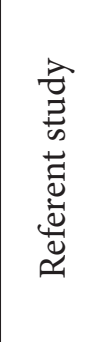 & 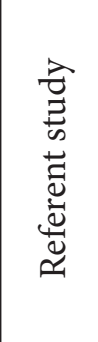 & 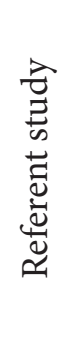 & 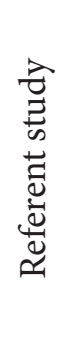 & 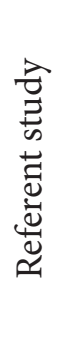 & 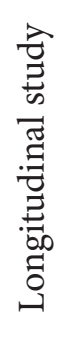 & 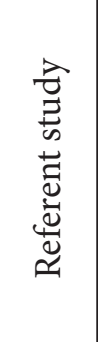 & 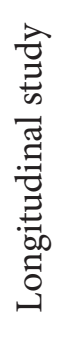 & 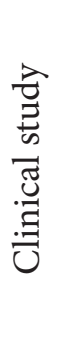 & 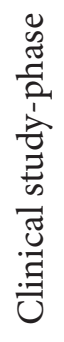 & 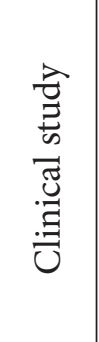 & 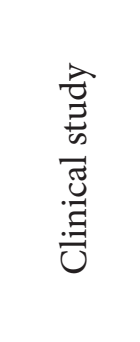 \\
\hline 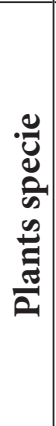 & 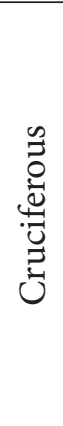 & 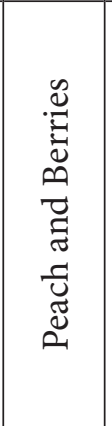 & 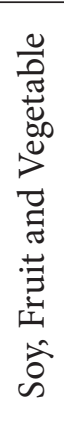 & ते & 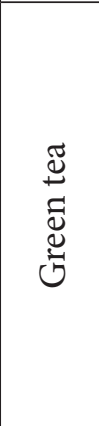 & $\stackrel{\Xi}{\oplus}$ & 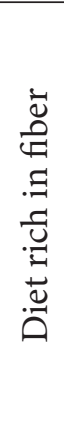 & 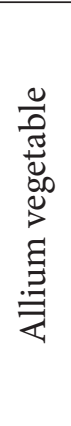 & 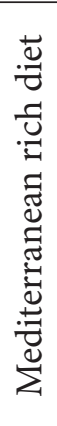 & 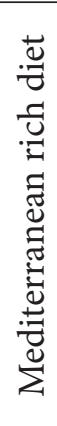 & 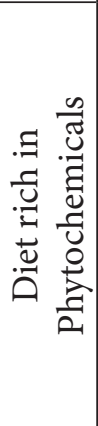 & 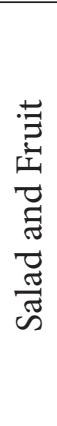 & 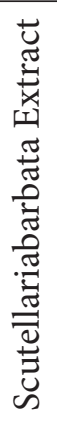 & 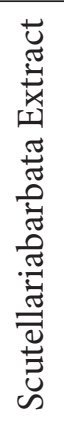 & 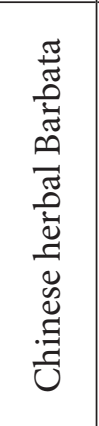 & 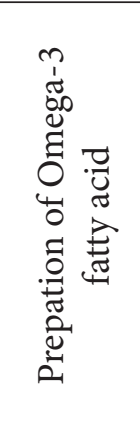 \\
\hline
\end{tabular}




\subsubsection{Causing Cell Apoptosis}

It has been reported that the programed cell death of cancer cells due to various phytochemicals is because of their ability to arrest the cell at G1-S and G2-M transition. Antioxidant effects and chemoprevention against cancers are exhibited by several phytochemicals ${ }^{61,62}$. Phyto nutrients affects both the intrinsic and extrinsic pathways of apoptosis mediated by their anticancer properties, activating caspase-3 which is downstream effector proteases (caspases) ${ }^{63,64}$. Quercetin, curcumin, tea flavonoids and genistein, have been shown to down regulate the signaling pathways of EGFR, ERK and Akt and activates JNK signaling which exhibits pro-apoptotic and anti-proliferative effect ${ }^{65,66}$. In addition to antioxidant properties majority of the phytochemicals are known to induce oxidative stress as well. Poly-phenolic compounds behave as both antioxidants and pro-oxidants depending upon the cellular concentration of $\mathrm{ROS}^{67}$. Usually, phytochemicals causes disproportion in the redox status of the cell by the production of ROS, which modifies the pathways of apoptosis through mitochondria and p38 MAP kinase ${ }^{68,69}$. As a result, the ROS generated by phytochemicals damages the DNA that leads to programed death of tumor cells ${ }^{70}$. This clearly suggests that several extracts of plant and phytochemicals exhibits inhibition of signal proteins or molecules associated with the survival, stress and death pathways of the cell.

\subsubsection{Angiogenesis}

Tumor growth results in the formation of new blood vessels from the pre-existing ones by angiogenesis. It is a critical step in the progression of tumor. Thus, angiogenesis has gained an attention as an important target in the treatment of several cancers. A monoclonal antibody known as Bevacizumab is the first FDA approved antiangiogenic drug. This drug targets the Vascular Endothelial Growth Factor A (VEGF-A), which is an essential factor for angiogenesis. There are more than 10 antiangiogenis drugs which are in clinical use nowadays. There are few natural compounds which have been reported as antiangiogenic compounds. Previous reviews by Mirossay et al. (2018) and Mojziset al. (2008) have discussed chalcones and flavonoids as the inhibitors of angiogenesis ${ }^{71,72}$. Moreover, some other phenolic compounds, including resveratrol, Gallic acid and curcumin also show antiangiogenic effects ${ }^{73,74}$. Several marine-derived compounds were reported to inhibit angiogenesis after extensive experimental research against cancer ${ }^{75}$. Acquired resistance results in the failure of treatment procedures in most of the cancer patients. In tumor mass a group of cells are present known as Cancer Stem Cells (CSC). These cells having aggressive phenotypes are thought to be responsible for the failure of cancer treatments such as chemotherapy, radiotherapy and immune therapy ${ }^{76,77}$. The maintenance and progression of tumor, recurrence of cancer and metastasis are due to the resistance in cancer treatment. Chemo-resistance can be overcome by targeting these cancer stem cells. This can be an effective strategy to treat cancer. The reported literature demonstrated that the signaling systems in CSCs which are involved in the self-renewal and proliferation of CSCs can be intervened by phytochemicals ${ }^{78-80}$. The use of phytochemicals poses a great strategy to eradicate CSCs. Thus, these molecules may help the researchers to develop novel drugs that can target and eradicate breast $\mathrm{CSCs}^{81}$.

\subsubsection{Epigenome}

According to the research, the sporadic breast cancer can be initiated and spread by epigenetic modifications. The risk of cancer development in an individual can be predicted, which is the fast growing field within clinical medicine. Research in oncology outlines the specific epigenetic aberrations, such as methylation status of tumor activating and suppressing genes, histones modification, and regulation noncoding RNA-linked genes $^{82,83}$. Epigenetic alterations and mutations in enzymes responsible for the regulation of chromatin may results in the unusual chemical modification of histones and alter the synthesis of histone modifying enzymes. Phytochemicals efficiently causes epigenetic changes and thus can play role in cancer chemoprevention ${ }^{83}$. Various in vivo and in vitro studies demonstrated the role of phytochemicals in the acetylation and methylation of histone proteins. Isolated phytochemicals or their combination alters the oncogenic or tumor suppressive miRNAs expression as demonstrated by numerous preclinical and few clinical studies on breast cancer cells ${ }^{84,85}$. The malignant related genes may get silenced by methylation. Hyper methylation of the promoter region of tumor suppressor genes plays a crucial in the development of cancer $^{86}$. The miRNA expression and DNA methylation along with better understanding of pathways involved in cancerous cellular signaling could serve in the establishment of effective chemo preventive and therapeutic approaches. For this purpose, several phytochemicals showed potential source to alter the epigenetics ${ }^{83}$. 


\subsection{Plant-derived Compounds Potential in Preclinical Breast Cancer Studies}

The anticancer activity of phytochemicals and their mode of action is evaluated by several preclinical and few clinical studies. Numerous plant derived compounds were assessed to describe their potential role in treating neoplastic disease, which findings may start a new potential site in cancer therapy research.

\subsubsection{In-Vitro Studies}

Phytochemicals could serve as a potential source to prevent and treat breast cancer and several other types of cancer. Origanum vulgare L., Chlorella pyrenoidosa, Syzygium aromaticum L., young barley grass, and fruit peel polyphenols were evaluated for their anticancer activities using an invite model of breast carcinoma. The antioxidant, antiviral, antimicrobial, anti-inflammatory properties of phytochemicals present in chlorella makes it an important functional food ${ }^{87,88}$. The in vitro study in human breast adenocarcinoma cells showed proapoptotic and anti-proliferative effects of Chlorella pyrenoidosa. The extract of Cholorella pyrenoidosa causes the ROS accumulation in MCF-7 cells. Results demonstrated that the proapoptosis caused by chlorella extracts is due to the production of ROS ${ }^{15}$. Another vitro study conducted by Kunte and Desai (2018) revealed that the extracts of Chlorella minutissimais involved in the inhibition of MMP-1, -2 , and -9 synthesis by interfering at both mRNA and protein level and causes inhibition of the active forms of MMP-2 and -9 in breast cancer model ${ }^{89}$. The extract of young barley, which is domesticated crop, contains many phenolic compounds such as leucoanthocyanidins, catechins, and flavones including 2-O-glucosylvitexin saponarin, coumarins and lutonarinn ${ }^{90}$. Evidence shows the anticancer activities of extracts of young barley ${ }^{91,92}$. Another study conducted on in vitro breast cancer model demonstrated that the young barley causes caspasedependent proapoptosis ${ }^{16}$. This effect is due to the arrest of cell cycle at $S$ phase, resulting into apoptosis. Furthermore, it was shown that the apoptotic activity of barley extracts is due to the accumulation of ROS in the breast cancer cells ${ }^{93}$. These results suggested that young barley could serve as a safe dietary agent in the cancer chemoprevention in BC models. Researchers are still trying to find out the possible mechanisms.

The volatile oil of an aromatic plant known as Origanum vulgare L. contains excessive amounts of phenolic compounds and monoterpenoids that exhibit antioxidant and antimicrobial activities $^{94,95}$. Recently, the in-vitro cancer lines were used to evaluate the ant proliferative effects of oregano crude extract ${ }^{96}$. Kubatka et al. demonstrated that oregano activates caspase- 7 and induces apoptosis in MCF-7 cells. Moreover, oregano induces apoptosis by the deactivation of protein $\mathrm{Bcl}-$ 218. Several studies showed interesting results in in vitro breast cancer model using Origanum majorana MDA-MB-231 BC cells were also assessed to determine the cytotoxic effects of $O$. majorona extracts ${ }^{97}$. Al Dhaheri et al. demonstrated the effects of O.majorona in the triple negative MDA-MB-231 cells ${ }^{98}$. They demonstrated that the cytotoxic activity is dependent on the concentrations of crude extract used. Low concentration off oregano crude extract induces mitotic arrest by damaging DNA and increases the expression of p21 and surviving which are CDK inhibitors.

Conversely, major apoptosis caused by the activating TNF-alpha extrinsic pathway was induced by high oregano concentrations. It is accompanied by the activation of caspase-8, high levels of the damage to DNA, depletion of p53 mutations, and, interestingly, deficiency of surviving proteins. Majorana also has antiinvasive and anti-metastatic effects against the cell line of breast cacner. Importantly, these effects are mediated by controlling cell migration, adhesion or invasion by modulating the activities or expression of proteins, such as MMP-9, E-cadherin, uPAR, MMP-2, VEGF and ICAM-1at least in part by inhibiting the signaling pathways of $\mathrm{NO}$ and $\mathrm{NF}^{-\mathrm{B}^{99}}$. Owing mainly to his antioxidant and antimicrobial activities, Clove attracted attention ${ }^{100}$. The anti-proliferative properties of the extracts of clove or eugenol have also been studied in the last decade. The strong anti-proliferative effect of these extracts was observed. The clove extract treated MCF-7 cells have been shown to contribute to cell aggregation in the $S$ process shortly after 24 hours. In addition, major cell augmentation in the sub-G0/G1 fraction was also detected. In addition, the dysregulation of mitochondria was associated with activation of caspase, inhibition of Bcl-2 activity, thus leads to the programmed death of the cell ${ }^{19}$. The anti-proliferative efficacy in five separate cancer cell lines was studied ${ }^{101}$. Later, in both cancer cell lines the growth inhibition by the extracts of clove was confirmed by Aisha et al. ${ }^{102}$

The anticancer effects of single flavonoids have been tested by several in vitro studies ${ }^{103,104}$. However, flavonoid combination has higher anticancer efficacy compared with a single flavonoid as shown by several studies. The polyphenols (Flavin 7) present in the fruit peel also inhibits the growth of MCF-7 cell by arresting the cell cycle at G1 phase ${ }^{17}$. Later it was found that the 
dysfunction of mitochondria accompanied by caspasedependent apoptosis was caused by Flavin-7. In addition, modulation was also demonstrated for signaling pathways. In addition, the role of ROS in cell death induced by Flavin-7 was also clearly established ${ }^{105}$. More recently, Kim et al. and Shanmugapriya et al. have also reported that ROS production in breast cancer cells by phenol rich plant extracts induces apoptosis of theses cell ${ }^{104,106}$. In MCF-7-treated cells, both authors revealed that cell death is associated with the increase in the production of ROS. Thus, many other functional plant foods with reported in vitro anti-cancer activities against breast cancer are also available ${ }^{107,108}$.

\subsubsection{In Vivo Studies}

In in vivo breast cancer models, plant-derived foods have proven anticancer properties. Several animal studies have shown that plant derived foods minimize the threat of experimental BC significantly. 7,12-Di-methylBenz(a) Anthracene (DMBA)-induced rat mammary carcinogenesis was inhibited effectively by the extracts of rosemary and its isolated component carnosol. Rosemary reduces the tumor frequency in each rat by $74 \%$ and carnosal did the similar reduction by $65 \%$ compared with controls. Moreover as compared to controls, rosemary as well as carnosol efficiently decreased the in vivo development of rat mammary DMBA-DNA adducts by $44 \%$ and $40 \%$ respectively 8 .

In other findings, it has been reported by Jeyabalan et al. that blueberries minimizes the occurrence of tumor of estradiol mammary carcinoma in female rats by $48 \%$ and $58 \%$ and also have therapeutic and chemopreventive mode 9 . Anticancer effects were due to the down-regulation of expression of genes ER- $\alpha$ and CYP1A1 and change in level of miRNA in mammary tissues. Adam et al. worked on xenograft model of MDA-MB-231 cells in female nude mice to evaluate the effects of blueberry powder in two different concentrations ( $5 \%$ and $10 \%)^{10}$. As a result blueberries showed significant reduction of tumor volume in which $5 \%$ showed $75 \%$ reduction and $10 \%$ exhibited $60 \%$ reduction as compared to controls. However, $25 \%$ less lymph node metastasis as compared to control was developed by the mice fed with $5 \%$ of the blueberries powder. After a high dose of therapy, blueberries showed significant anti-proliferative activity in treated groups. It also altered the expression of IL-13, IFN $\gamma$ and Wnt thrombospondin-2. The anticancer properties of black and blue berries were compared by Ravoori et al. in rat mammary oncogenesis mediated by estrogen ${ }^{11}$. Both significantly prolonged the expectancy of tumor by 24 and
39 days, respectively. Moreover, blueberries significantly reduced the tumor frequency and proliferation of mammary tissues. Blueberries down-regulate the CYP1A expressions while blackberries down-regulate the ERa expression meritoriously. Isoflavone-rich soy germ effectively reduced the development of mammary cancer in rats induced by $\mathrm{PhIP}$. Histopathologically, it has been verified that less malignant and non-fibrous features of carcinoma treated with soy germ ${ }^{109}$. Another study revealed that by $17 \beta$-estradiol of caraway significantly delayed and prevented tumerogenesis in rat. It has been described that with caraway in diet reduced tumor frequency by $40 \%$ and tumor volume by $53 \%$ in treated animals versus control. Caraway down-regulate the expression of estrogen receptor $\alpha$, cyclin D1, CYP1B1 and CYP1A1. Bishayee et al. demonstrated that pomegranate emulsion showed outstanding chemopreventive activity in induced-DMBA mammary oncogenesis in rat ${ }^{13}$. Different quantities $(0.2,1,5 \mathrm{~g} / \mathrm{kg})$ of pomegranate reduced the tumor frequency, tumor weight, tumor burden as compared to controls. Pomegranate specifically enhanced the expressions of caspase-3/-7/-9, Bad, Bax/bcl-2 ratio, cytochrome $c$ and poly (ADP ribose) polymerase, and showed antiapoptotic and anti-proliferative activities in the cancer tissues of rat. In addition, the same authors ${ }^{12}$ revealed that pomegranate decreased the expression of intratumor ER- $\alpha$ and ER- $\beta$ and reduced the ER- $\alpha$ : ER- $\beta$ ratio. PE silenced the expression of cyclin D1 and Wnt signaling (via $\beta$-catenin) in mammary carcinomas of rat induced by DMBA. In another study, the extracts of Cyclopiasubternata reduced the volume of mammary tumor at the same rate as tamoxifen in an orthotopic rat model. Notably, in animals no side effects of tamoxifen and letrozole were reported ${ }^{110}$. The in vivo anticancer effects of plant derived foods against mammary carcinoma were also demonstrated by several recently published papers $^{14,111}$. Recently, the chemo-preventive efficacy of numerous plant derived foods against rat mammary carcinogenesis was also demonstrated. Origanumvulgare L. $(0.1 \%)$ administered in diet effectively inhibited the tumor frequency by $55.5 \%$, repressed tumor occurrence by $44 \%$, and decreases the volume of tumor mass by $44.5 \%$ in comparison to control group ${ }^{18}$. The expression of VEGFR-2, CD24, EpCam, and Ki67 has been shown to down regulate in rat cancer cells. On the other hand, same dosage of oregano increased the caspase- 3 expression. $3 \%$ oregano increased the tumor latency by 12.5 days. Moreover, in in vivo cancer cells the expression of CD24, VEGFR-2, Bcl-2, and EpCam got down-regulated and the expression of caspase- 3 has been up regulated. Histopathalogial analysis showed that the tumor ratio in 
comparison to control group was decreased by the use of oragano. In another study, the chemo-preventive effects against mammary carcinogenesis in rats were analyzed by providing $0.1 \%$ and $1 \%$ of Syzygium aromaticum $\mathrm{L}$. in $\operatorname{diet}^{19}$. According to the results, the tumor frequency was suppressed by $47.5 \%$ and $58.5 \%$ by administering clove buds in dose dependent manner as compared with control. Immunohistochemical analysis of carcinoma cells demonstrated that high dose of clove administration was linked with the down regulation in expression of Ki67, Bcl-2, and VEGFA, CD24 and CD44. ${ }^{19}$

Similarly, the same group showed enhanced expression of ALDH 1, caspase-3 and Bax. Besides, in both clove groups the level of MDA was decreased considerably in mammary tumors. After oregano treatment, the analyses of histone modifications revealed that trimethylations and acetylations of lysine were increased in carcinomas. Clove administration in rats did not show any considerable changes in methylation of promoter of RASSF1A and TIMP3. Our experimental findings $(0.3 \%$ and $3 \%$ in the diet $)$ with dark fruit peels revealed that frequency of tumor decreased by $58 \%$ as compared with control. Moreover, it has also been demonstrated that latency was lengthened by 8 days and tumor frequency was also decreased by $24 \%$ as compared with control. In higher and low dose group carcinomas, its chemoprevention feature showed significantly decreased in ratio. Additionally, the analysis of rat carcinoma cells demonstrated the increased expression of caspase- 3 and decrease in the VEGFR-2, Bcl-2, and Ki67 expression by the intake of dark fruit peels in high-dose ${ }^{17}$. However, the same breast cancer rat models were used to test the anticancer efficacy of the two green foods i.e. young barley grass and Chlorella pyrenoidosa (doses of $0.3 \%$ and $3 \%$ in the diet). It was observed that Chlorella reduced the frequency of tumor by $61 \%$ and extends the tumor latency by 12.5 days as compared with control group ${ }^{15}$. Higher dose of chlorella increases the expression of caspase- 7 by $73.5 \%$ and decreases the expression of vascular endothelial growth factor receptor-2 by $19 \%$ in rat tumor cells. Only minute antitumor effect of $0.3 \%$ barley was observed in carcinomas and no improvements were detected by increasing the dose of barley. The treated groups showed enhanced expression of caspase-3 and reduced expression of Ki67 as revealed by Immunohistochemical analysis tumor cells in rats. ${ }^{15}$

\section{Conclusions}

The critical assessment of the literature showed a wide range of the anticancer effects of herbal compounds as synergistic or additive effects against breast cancer that could aid in designing specified treatment strategies. However, there is lack of literature showing the validation of these findings in clinical trials. Therefore, the animal studies and clinical trials are needed to be conducted for the evaluation of these herbal compounds and make them a step closer towards potential therapeutic strategy against breast cancer. The anti-neoplastic efficiency of these herbal compounds is sometimes overlaid by many unidentified high-risk factors in females, which are not considered in laboratory studies. Thus, more elaborative studies, considering well-designed and strictly controlled research conditions and clinical trials are needed in this regard. The adverse effects of herbal compounds in cancer patients could be attributed to the inappropriate use of drugs combination. Additionally, the herbal compounds can also promote tumor or act as carcinogens. Therefore, given the inappropriate interference of herbal compounds in the treatment of cancer, it is highly recommended to be more careful in patients having hormonal or target therapy.

\section{Acknowledgements}

None

\section{References}

1. Golubnitschaja O, Debald M, Yeghiazaryan K, Kuhn W, Pes `ta M, Costigliola V, et al. Breast cancer epide micin the early $21^{\text {st }}$ century: evaluation of risk factors, cumulative questionnaires and recommendations for preventive measures. Tumor Biol. 2016;37(10):1294112957. https://doi.org/10.1007/s13277-016-5168-x. PMid:27448308.

2. Liu RH. Dietary bioactive compounds and their health implications. J Food Sci 2013;78(s1):A18-A25. https:// doi.org/10.1111/1750-3841.12101. PMid:23789932.

3. Liu RH. Health-promoting components of fruits and vegetables in the diet. Adv Nutr. 2013;4(3):384S-392S. https://doi.org/10.3945/an.112.003517. PMid:236748 08 PMCid:PMC3650511.

4. Yiannakopoulou EC. Effect of green tea catechins on breast carcinogenesis: a systematic review of in-vitro and in-vivo experimental studies. Eur J Cancer Prev. 2014;23(2):84-89.https://doi.org/10.1097/CEJ.0b013e 328364f23e. PMid:23939462.

5. Kapinova A, Stefanicka P, Kubatka P, Zubor P, Uramova S, Kello M, et al. Are plant-based functional foods better choice against cancer than single phyto 
chemicals? A critical review of current breast cancer research. BiomedPharmacother. 2017;96:1465-1477. https://doi.org/10.1016/j.biopha.2017.11.134. PMid:2 9198744.

6. Kulkarni AD, Sundaresan A, Rashid MJ, Yamamoto S, Karkow F. Application of diet-derived taste active components for clinical nutrition: perspectives from ancient Ayurvedic medical science, space medicine, and modern clinical nutrition. Curr Pharm Des. 2014;20(16):2791-2796. https://doi.org/10.2174/1381 6128113199990571 . PMid:23886389.

7. Wang CY, Bai XY, Wang CH. Traditional Chinese medicine: a treasured natural resource of anticancer drug research and development. Am J Chin Med. 2014;42(03):543-559. https://doi.org/10.1142/ S0192415X14500359. PMid:24871650.

8. Singletary K, MacDonald C, Wallig M. Inhibition by rosemary and carnosol of 7,12dimethylbenz [a] anthracene (DMBA)-induced rat mammary tumor igenesis and in vivo DMBA-DNA adduct forma-tion. Cancer Lett. 1996;104(1):43-48. https://doi.org/10.10 16/0304-3835(96)04227-9.

9. Jeyabalan J, Aqil F, Munagala R, Annamalai L, Vadhanam MV, Gupta RC. Chemopreventive and therapeuticactivity of dietaryblueberry against estrogen-mediated breast cancer. J Agric Food Chem. 2014;62(18):3963-3971. https://doi.org/10.1021/jf403 734j. PMid:24245576 PMCid:PMC4334276.

10. Adams LS, Kanaya N, Phung S, Liu Z, Chen S. Whole blueberry powder modulates growth and metastasis of MDA-MB-231 triple negative breast tumors in nude mice. J Nutr. 2011;141(10):1805-1812. https:// doi.org/10.3945/jn.111.140178. PMid:21880954 PMC id:PMC3174855.

11. Ravoori S, Vadhanam MV, Aqil F, Gupta RC. Inhibition of estrogen-mediated mammary tumorigenesis by blueberry and black raspberry. J Agric Food Chem. 2012;60(22):5547-5555. https://doi. org/10.1021/jf205325p. PMid:22571764.

12. Mandal A, Bishayee A. Mechanism of breast cancer preventive action of pomegranate: disruption of estrogen receptor and Wnt $/ \beta$-catenin signaling pathways. Molecules. 2015;20(12):22315-22328. https://doi.org/10.3390/molecules201219853. PMid:2 6703530 PMCid:PMC6332439.

13. Bishayee A, Mandal A, Bhattacharyya P, Bhatia D. Pomegranate exerts chemoprevention of experi mentally induced mammary tumorigenesis by suppression of cell proliferation and induction of apoptosis. Nutr Cancer. 2015;68(1):120-130. https:// doi.org/10.1080/01635581.2016.1115094. PMid:2669 9876 PMCid:PMC4784500.

14. Nassan MA, Soliman MM, Ismail SA, El-Shazly SA. Effect of Taraxacumofficinale extract on PI3K/Akt pathway in DMBA induced breast cancer in albino rats. Biosci Rep. 2018;38(6). https://doi.org/10.1042/ BSR20180334. PMid:30126855 PMCid:PMC6435453.

15. Kubatka P, Kapinová A, Kružliak P, Kello M, Výbohová D, Kajo K, et al. Antineoplastic effects of Chlorella pyrenoidosa in the breast cancer model. Nutrition. 2015; 31(4):560-569. https://doi.org/10.1016/j.nut.2014.08.010. PMid:25770318.

16. Kubatka P, Kello M, Kajo K, Kruzliak P, Výbohová $\mathrm{D}$, Smejkal K, et al. Young barley indicates antitumor effects in experimental breast cancer in vivo and in vitro. Nutr Cancer. 2016;68(4):611-621.https://doi.org /10.1080/01635581.2016.1154577. PMid:27042893.

17. Kubatka P, Kapinová A, Kello M, Kruzliak P, Kajo K, Výbohová D. et al, Fruit peel polyphenols demonstrate substantial anti-tumour effects in the model of breast cancer. Eur J Nutr. 2016;55(3):955-965. https://doi. org/10.1007/s00394-015-0910-5. PMid:25930965.

18. Kubatka P, Kello M, Kajo K, Kruzliak P, Výbohová $\mathrm{D}$, Mojžiš J, et al., Oregano demonstrates distinct tumour-suppressive effects in the breast carcinoma model. Eur J Nutr. 2017;56(3):1303-1316. https://doi. org/10.1007/s00394-016-1181-5. PMid:26907089.

19. Kubatka, P., Uramova, S., Kello, M., Kajo, K., Kruzliak, P., Mojzis, J., et al., Antineoplastic effects of clove buds (Syzygiumaromaticum L.) in the model of breast carcinoma. J Cell Mol Med. 2017;21(11):2837-2851. https://doi.org/10.1111/jcmm.13197. PMid:28524540 PMCid:PMC5661249.

20. Liu X, Lv K. Cruciferous vegetables intake is inversely associated with risk of breast cancer: a meta-analysis. Breast. 2013;22(3):309-313. https://doi.org/10.1016/j. breast.2012.07.013. PMid:22877795.

21. Fung TT, Chiuve SE, WillettWC, Hankinson SE, Hu FB, Holmes MD. Intake of specific fruits and vegetables in relation to risk of estrogen receptor-negative breast cancer among postmenopausal women. Breast Cancer Res Treat. 2013;138(3):925-930. https://doi. org/10.1007/s10549-013-2484-3. PMid:23532538 PM Cid:PMC3641647.

22. Butler LM, Wu AH, Wang R, Koh WP, Yuan JM, Yu MC. A vegetable-fruit-soy dietary pattern protects against 
breast cancer among postmenopausal Singapore Chinese women. Am J Clin Nutr. 2010;91(4):10131019. https://doi.org/10.3945/ajcn.2009.28572. PM id:20181808 PMCid:PMC2844682.

23. Ko KP, Kim SW, Ma SH, Park B, Ahn Y, Lee JW, et al. Dietary intake and breast cancer among carriers and noncarriers of BRCA mutations in the Korean Hereditary Breast Cancer Study. Am J Clin Nutr. 2013;98(6):1493-1501. https://doi.org/10.3945/ajcn.1 12.057760. PMid:24153343.

24. Li M, Tse LA, Chan WC, Kwok CH, Leung SL, Wu C, et al. Evaluation of breast cancer risk associated with tea consumption by menopausal and estrogen receptor status among Chinese women in Hong Kong. Cancer Epidemiol. 2016;40:73-78. https://doi.org/10.1016/j. canep.2015.11.013. PMid:26680603 PMCid:PMC671 9777.

25. Kumar N, Titus-Ernstoff L, Newcomb PA, Tren tham-Dietz A, Anic G, Egan KM. Tea consumption and risk of breast cancer. Cancer Epidemiol Biomarkers Prev. 2009;18(1):341-345. https://doi. org/10.1158/1055-9965.EPI-08-0819. PMid:1912451 8 PMCid:PMC3156033.

26. Sangaramoorthy M, Koo J, John EM. Intake of bean fiber, beans, and grains and reduced risk of hormone receptor-negative breast cancer: The San Francisco Bay Area Breast Cancer Study. Cancer Med. 2018; 7(5):2131-2144. https://doi.org/10.1002/cam4.1423. PMid:29573201 PMCid:PMC5943543.

27. Pourzand A, Tajaddini A, Pirouzpanah S, AsghariJafarabadi M, Samadi N, Ostadrahimi AR, et al. Associationsbetween dietaryalliumvegetablesand risk ofbreastcancer: a hospital-based matched case-control study. Breast Cancer. 2016;19(3), 292-300. https://doi. org/10.4048/jbc.2016.19.3.292. PMid:27721879 PMC id:PMC5053314.

28. Castello' A, Polla'n M, Buijsse B, Ruiz A, Casas AM, Baena-Can ada JM, et al. Spanish Mediter-ranean diet and other dietary patterns and breast cancer risk: Case-control EpiGEICAM study. Br J Cancer. 2014; 111(7):1454-1462. https://doi.org/10.1038/bjc. 2014.434. PMid:25101568 PMCid:PMC4183855.

29. Cottet V, Touvier M, Fournier A, Touillaud MS, Lafay L, Clavel-Chapelon F, etal.Postmenopausal breastcancer risk and dietary patterns in the E3N-EPIC prospective cohort study. Am J Epidemiol. 2009; 170(10):12571267. https://doi.org/10.1093/aje/kwp257. PMid:1982 8509.
30. Bahadoran Z, Mirmiran P, Azizi F. Dietary polyphenols as potential nutraceuticals in management of diabetes: A review. J Diabetes MetabDisord. 2013;12(1):43. https://doi.org/10.1186/2251-6581-12-43. PMid:2393 8049.

31. Baglietto L, Krishnan K, Severi G, Hodge A, Brinkman $\mathrm{M}$, English DR, et al. Dietary patterns and risk of breast cancer. Br J Cancer. 2011;104(3):524-31. https://doi. org/10.1038/sj.bjc.6606044. PMid:21157446 PMCid: PMC3049555.

32. Rugo H, Shtivelman E, Perez A, Vogel C, Franco S, Tan Chiu E, et al. Phase I trial and antitumor effects of BZL101 for patients with advanced breast cancer. Breast Cancer Res Treat. 2007;105(1):17-28. https:// doi.org/10.1007/s10549-006-9430-6. PMid:17111207.

33. Perez AT, Arun B, Tripathy D, Tagliaferri MA, Shaw HS, Kimmick GG, et al. A phase 1B dose escalation trial of Scutellariabarbata (BZL101) for patients with metastatic breast cancer. Breast Cancer Res Treat. 2010;120(1):111-118. https://doi.org/10.1007/ s10549-009-0678-5. PMid:20054647.

34. Mao Y, Hao J, Jin ZQ, Niu YY, Yang X, Liu D, et al. Network pharmacology-based and clinically relevant prediction of theactiveingredientsand potential targets of Chinese herbs in metastatic breast cancer patients. Oncotarget. 2017;8(16):27007-27021. https://doi. org/10.18632/oncotarget.15351. PMid:28212580 PM Cid:PMC5432314.

35. Manni A, Richie JP, Schetter SE, Calcagnotto A, Trushin N, Aliaga C, et al. Stearoyl-CoA desaturase-1, a novel target of omega-3 fatty acids for reducing breast cancer risk in obese postmenopausal women. Eur J ClinNutr. 2017; 71(6):762-765. https://doi.org/ 10.1038/ejcn.2016.273. PMid:28145413.

36. Zou M, Xu C, Li H, Zhang X, Fan W. 3,3'-Diindoly lmethane suppresses ovarian cancer cell viability and metastasis and enhances chemotherapy sensitivity via STAT3 and Akt signaling in vitro and in vivo. Arch BiochemBiophys. 2018; S0003-9861(18):3008730090. https://doi.org/10.1016/j.abb.2018.07.002.

37. Park S, Cho DH, Andera L, Suh N, Kim I. Curcumin enhances TRAIL-induced apoptosis of breast cancer cells by regulating apoptosis-related proteins. Mol Cell Biochem. 2013;383(1-2):39-48. https://doi.org/ 10.1007/s11010-013-1752-1. PMid:23846485.

38. Manouchehri JM, Turner KA, Kalafatis M. TRAILInduced Apoptosis in TRAIL-Resistant Breast Carc inoma Through Quercet in Cotreatment. Breast 
Cancer (Auckl). 2018;12:1-12. https://doi.org/10. 1177/1178223417749855. PMid:29434473 PMCid:P MC5802616.

39. Tseng HS, Wang YF, Tzeng YM, Chen DR, Liao YF, Chiu HY, et al. Aloe-Emodin Enhances Tamoxifen Cytotoxicity by Suppressing Ras/ERK and PI3K/ mTOR in Breast Cancer Cells. Am J Chin Med. 2017;45(2):337-350. https://doi.org/10.1142/S019241 5X17500215. PMid:28231748.

40. Diaz-Chavez J, Fonseca-Sanchez MA, ArechagaOcampo E, Flores-Perez A, Palacios-Rodriguez Y, Dominguez-Gomez G, et al. Proteomic profiling reveals that resveratrol inhibits HSP27 expression and sensitizes breast cancer cells to doxorubicin therapy. PLoS One. 2013;8(5):e64378. https://doi.org/10.1371/ journal.pone.0064378. PMid:23724044 PMCid:PMC 3664632 .

41. Ito Y, Mitani T, Harada N, Isayama A, Tanimori S, Takenaka S, et al. Identification of carbonyl reductase 1 as a resveratrol-binding protein by affinity chromatography using 4'-amino-3,5-dihydroxy-trans-stilbene. J Nutr Sci Vitaminol (Tokyo). 2013; 59(4):358-364. https://doi. org/10.3177/jnsv.59.358. PMid:24064738.

42. Richards CE, Vellanki SH, Smith YE, Hopkins AM. Diterpenoid natural compound C4 (Crassin) exerts cytostatic effects on triple-negative breast cancer cells via a pathway involving reactive oxygen species. Cell Oncol (Dordr). 2018; 41(1):35-46. https://doi. org/10.1007/s13402-017-0357-1. PMid:29134467.

43. Di Y, De Silva F, Krol ES, Alcorn J. Flaxseed Lignans Enhance the Cytotoxicity of Chemotherapeutic Agents against Breast Cancer Cell Lines MDA-MB-231 and SKBR3. Nutr Cancer. 2018; 70(2):306-315. https://doi.org/10.1080/01635581.2018.1421677. PMid:29303360.

44. Mundhe NA, Kumar P, Ahmed S, Jamdade V, Mundhe S, Lahkar M. Nordihydroguaiaretic acid ameliorates cisplatin induced nephrotoxicity and potentiates its anti-tumor activity in DMBA induced breast cancer in female Sprague-Dawley rats. Int. Immunopharmacol. 2015; 28(1):634-642. https://doi. org/10.1016/j.intimp.2015.07.016. PMid:26247680.

45. Hanušová V, Caltová K, Svobodová $H$, Ambrož $\mathrm{M}$, Skarka A, Murínová $\mathrm{N}$ et al. The effects of $\beta$-caryophyllene oxide and trans -nerolidol on the efficacy of doxorubicin in breast cancer cells and breast tumor-bearing mice. Biomed Pharmacother. 2017;95:828-836. https://doi.org/10.1016/j.biopha.20 17.09.008. PMid:28903178.
46. Zhong Y, Zhang F, Sun Z, Zhou W, Li ZY, You QD, et al. Drug resistance associates with activation of Nrf2 in MCF-7/DOX cells, and wogonin reverses it by downregulating Nrf2-mediated cellular defense response. MolCarcinog. 2013; 52(10):824-834. https://doi.org/1 0.1002/mc.21921. PMid:22593043.

47. Zhang S, Sagawa K, Arnold RD, Tseng E, Wang $\mathrm{X}$, Morris ME. Interactions between the flavonoid biochanin A and P-glycoprotein substrates in rats: In vitro and in vivo. J Pharm Sci. 2010;99(1):430-441. https://doi.org/10.1002/jps.21827. PMid:19499569.

48. Qian J, Xia M, Liu W, Li L, Yang J, Mei Y, et al. Glabr idinresensitizes p-glycoprotein-overexpressing multi drug-resistant cancer cells to conventional chemo therapeutic agents. Eur J Pharmacol. 2019;852:231243. https://doi.org/10.1016/j.ejphar.2019.04.002. PM id:30959046.

49. Zong L, Cheng G, Liu S, Pi Z, Liu Z, Song F. Reversal of multidrug resistance in breast cancer cells by a combination of ursolic acid with doxorubicin. J Pharm Biomed Anal. 2019;165:268-275. https://doi. org/10.1016/j.jpba.2018.11.057. PMid:30572191.

50. Louisa M,SoediroTM, SuyatnaFD. Invitromodulation of P-glycoprotein, MRP-1 and BCRP expression by mangiferin in doxorubicin-treated MCF-7 cells. Asian Pac J Cancer Prev. 2014; 15(4):1639-1642. https:// doi.org/10.7314/APJCP.2014.15.4.1639. PMid:24641 381.

51. Li YH, Niu YB, Sun Y, Zhang F, Liu CX, Fan L, Mei QB. Role of phytochemicals in colorectal cancer prevention. World J Gastroenterol. 2015;21(31):92629272. https://doi.org/10.3748/wjg.v21.i31.9262. PM id:26309353 PMCid:PMC4541379.

52. Geng Y, Zhou Y, Wu S, Hu Y, Lin K, Wang Y, et al. Sulforaphane induced apoptosis via promotion of mitochondrial fusion and ERK1/2-mediated 26S proteasome degrada-tion of novel pro-survival bim and upregulation of bax in human non-small cell lung cancer cells. J Cancer. 2017;8(13):2456-2470. https:// doi.org/10.7150/jca.19383. PMid:28900483 PMCid:P MC5595075.

53. Singh SK, Banerjee S, Acosta EP, Lillard JW, Singh R. Resveratrol induces cell cycle arrest and apoptosis with docetaxel in prostate cancer cells via a p53/ p21WAF1/CIP1 and p27KIP1 pathway. Oncotarget. 2017;8(10):17216-17228. https://doi.org/10.18632/ oncotarget.15303.PMid:28212547PMCid:PMC53700 34. 
54. Agarwal A, Kasinathan A, Ganesan R, Balasub ramanian A, Bhaskaran J, Suresh S, et al. Curcumin induces apoptosis and cell cycle arrest via the activation of reactive oxygen species-independent mitochondrial apoptotic pathway in Smad4 and p53 mutated colon adenocarcinoma HT29 cells. Nutr Res. 2018;51:67-81. https://doi.org/10.1016/j. nutres.2017.12.011. PMid:29673545.

55. Zhang Y, Chen S, Wei C, Rankin GO, Ye X, Chen YC. Flavonoids from Chinese bayberry leaves induced apoptosis and G1 cell cycle arrest via Erk pathway in ovarian cancer cells. Eur J Med Chem. 2018; 147:218226. https://doi.org/10.1016/j.ejmech.2018.01.084. PMid:29438890 PMCid:PMC5823286.

56. SabarwalA,AgarwalR,SinghRP.Fisetininhibitscellular proliferation and induces mitochondria-dependent apoptosis in human gastric cancer cells. MolCar cinog. 2017;56(2):499-514. https://doi.org/10.1002/ mc.22512. PMid:27254419.

57. Cheng YM, Tsai CC, Hsu YC. Sulforaphane, a dietary isothiocyanate, induces G2/M arrest in cervical cancer cells through cyclinB1 downregulation and GADD45 $\beta / C D C 2$ association. Int J MolSci. 2016;17(9):1530. https://doi.org/10.3390/ijms17091530. PMid:276264 12 PMCid:PMC5037805.

58. Zhang L, Cheng X, Gao Y, Bao J, Guan H, Lu R, et al. Induction of ROS-independent DNA damage by curcumin leads to G2/M cell cycle arrest and apoptosis in human papillary thyroid carcinoma BCPAP cells. Food Funct. 2016;7(1):315-325. https:// doi.org/10.1039/C5FO00681C. PMid:26442630.

59. Jeong JW, Park S, Park C, Chang YC, Moon DO, Kim SO, et al. N-benzyl-N-methyldecan-1-amine, a phenylamine derivative isolated from garlic cloves, induces G2/M phase arrest and apoptosis in U937 human leukemia cells. Oncol Rep. 2014;32(10):373381. https://doi.org/10.3892/or.2014.3215. PMid:248 59825.

60. Zhang Z, Wang CZ, Du GJ, Qi LW, Calway T, He TC, et al. Genistein induces G2/M cell cycle arrest and apoptosis via ATM/p53-dependent pathway in human colon cancer cells. Int J Oncol. 2013;43(1):289296. https://doi.org/10.3892/ijo.2013.1946. PMid:236 86257 PMCid:PMC3742162.

61. Chikara S, Nagaprashantha LD, Singhal J, Horne D, Awasthi S, Singhal SS. Oxidative stress and dietary phytochemicals: role in cancer chemoprevention and treatment. Cancer Lett. 2018;413:122-134. https://doi. org/10.1016/j.canlet.2017.11.002. PMid:29113871.
62. Hwang KA, Choi KC. Anticarcinogenic effects of dietary phytoestrogens and their chemopreven-tive mechanisms. Nutr Cancer. 2015; 67(5):796-803. https://doi.org/10.1080/01635581.2015.1040516. PMid:25996655.

63. Lou M, Zhang LN, Ji PG, Feng FQ, Liu JH, Yang C, et al. Quercetin nanoparticles induced autophagy and apoptosis through AKT/ERK/Caspase-3 signaling pathway in human neuro-glioma cells: in vitro and in vivo. Biomed Pharmacother. 2016;84:1-9. https://doi. org/10.1016/j.biopha.2016.08.055. PMid:27621033.

64. Okubo S, Uto T, Goto A, Tanaka H, Nishioku T, Yamada K, et al. Berberine induces apoptotic cell death via activation of caspase- 3 and- 8 in HL-60 human leukemia cells: nuclear local-ization and structure-acti vity relationships. Am J Chin Med. 2017;45(7):14971511. https://doi.org/10.1142/S0192415X17500811. PMid:29025293.

65. Yang Y, Wang T, Chen D, Ma Q, Zheng Y, Liao S, et al. Quercetin preferentially induces apoptosis in KRASmutant colorectal cancer cells via JNK signaling pathways. Cell Biol 2018;43(2):117-124. https://doi. org/10.1002/cbin.11055. PMid:30203888.

66. Satonaka H, Ishida K, Takai M, Koide R, Shigemasa R, Ueyama J, et al. (-)-Epigallocatechin-3-gallate downregulates doxorubicin-induced overexpres-sion of P-glycoprotein through the coordinate inhibition of PI3K/Akt and MEK/ERK signaling path-ways. Anticancer Res. 2017; 37(11):6071-6077. https://doi. org/10.21873/anticanres.12055.

67. Alrawaiq N, Abdullah A. Dietary phytochemicals activate the redox-sensitive transcription factor $\mathrm{Nrf} 2$. Int J Pharm PharmSci. 2014;6:11-16.

68. Chen JC, Hsieh MC, Lin SH, Lin CC, Hsi YT, Lo YS, et al. Coronarin $\mathrm{D}$ induces reactive oxygen speciesmediated cell death in human nasopharyngeal cancer cells through inhibition of p38 MAPK and activation of JNK. Oncotarget. 2017;8(64):108006108019. https://doi.org/10.18632/oncotarget.22444. PMid:29296219 PMCid:PMC5746121.

69. Chen XX, Lam KH, Chen QX, Leung GP, Tang SCW, Sze SC, et al. Ficusvirensproanthocyanidins induced apoptosis in breast cancer cells concomitantly ameliorated 5-fluorouracil induced intestinal mucositis in rats. Food ChemToxicol. 2017;110:49-61. https://doi.org/10.1016/j.fct.2017.10.017. PMid:2903 0256. 
70. Azqueta A, Collins A. Polyphenols and DNA damage: A mixed blessing. Nutrients. 2016;8(12):785. https://doi.org/10.3390/nu8120785. PMid:27918471 PMCid:PMC5188440.

71. Mirossay L, Varinska, L, Mojz ${ }^{\vee}$ is $^{\vee}$ J. Antiangiogenic effect of flavonoids and chalcones: an update. Int J MolSci. 2017; 19(1):27. https://doi.org/10.3390/ ijms19010027. PMid:29271940 PMCid:PMC5795978.

72. Mojzis J, Varinska L, Mojzisova G, Kostova I, Mirossay L. Antiangiogenic effects of flavonoids and chalcones. Pharmacol Res. 2008;57(4):259-265. https://doi. org/10.1016/j.phrs.2008.02.005. PMid:18387817.

73. Wong JC, Fiscus RR. Resveratrol at anti-angiogenesis/ anticancer concentrations suppresses pro-tein kinase $\mathrm{G}$ signaling and decreases IAPs expression in HUVECs. Anticancer Res. 2015; 35(1):273-281.

74. Varinska L, Mirossay L, Mojzisova G, Mojzis J. Antiangogenic effect of selected phytochemicals. Pharmazie. 2010;65(1):57-63.

75. Varinska L, Kubatka P, Mojzis J, Zulli A, Gazdikova K, ZuborP, etal.Angio-modulatorsincancer therapy:new perspectives. Biomed. Pharmacother. 2017;89:578590.https://doi.org/10.1016/j.biopha.2017.02.071.PM id:28258040.

76. Park SY, Lee HE, Li H, Shipitsin M, Gelman R, Polyak K. Heterogeneity for stemcell-related markers according to tumor subtype and histologic stage in breast cancer. Clin Cancer Res. 2010;16(3):876-887. https://doi.org/10.1158/1078-0432.CCR-09-1532. PMid:20103682 PMCid:PMC2818503.

77. Klonisch T, Wiechec E, Hombach-Klonisch S, Ande SR, Wesselborg S, Schulze-Osthoff K, et al. Cancer stem cellmarkers in common cancers-therapeutic implications. Trends Mol Med. 2008;14(10):450460. https://doi.org/10.1016/j.molmed.2008.08.003. PMid:18775674.

78. Choi HS, Kim SL, Kim JH, Deng, HY, Yun BS, Lee DS. Triterpene Acid (3-O-p-coumaroyltormentic acid) isolated from aronia extracts inhibits breast cancerstem cell formation through downregulation of c-myc protein. Int J MolSci. 2018;19(9):25-28. https:// doi.org/10.3390/ijms19092528. PMid:30149665 PMCid:PMC6164992.

79. Soltanian S, Riahirad H, Pabarja A, Jafari E, Khandani BK. Effect of Cinnamic acid and FOLFOX in diminishing side population and downregulating cancer stem cell markers in colon cancer cell line HT-29. DARU.
2018;26(1):19-29. https://doi.org/10.1007/s40199-0180210-8. PMid:30209760 PMCid:PMC6154487.

80. Aliebrahimi S, Kouhsari SM, Arab SS, Shadboorestan A, Ostad SN. Phytochemicals, witha-ferin A and carnosol, overcome pancreatic cancer stem cells as c-Met inhibitors. Biomed Pharmacother. 2018;106:1527-1536. https://doi.org/10.1016/j.bioph a.2018.07.055. PMid:30119228.

81. Hermawan A, Putri H. Current report of natural product development against breast cancer stem cells. Int J Biochem Cell Biol. 2018;104:114-132. https://doi. org/10.1016/j.biocel.2018.09.012. PMid:30266524.

82. Khan S, Shukla S, Sinha S, Meeran SM. Epigenetic targets in cancer and aging: dietary and therapeutic interventions. Expert OpinTher Targets. 2016;20(6):689-703. https://doi.org/10.1517/1472822 2.2016.1132702. PMid:26667209.

83. Uramova S, Kubatka P, Dankova Z, Kapinova A, Zolakova B, Samec M, et al. Plant natural modulators in breast cancer prevention: status quo and future perspectives reinforced by predictive, preventive and personalised medical approach. EPMA J. 2018;9(4):403-419. https://doi.org/10.1007/s13167018-0154-6. PMid:30538792 PMCid:PMC6261910.

84. Dagdemir A, Durif J, Ngollo M, Bignon YJ, BernardGallon D. Histone lysine trimethylation or acetylation can be modulated by phytoestrogen, estrogen or antiHDAC in breast cancer cell lines. Epigenomics. 2013; 5(1):51-63. https://doi.org/10.2217/epi.12.74. PMid:2 3414320 .

85. Attoub S, Hassan AH, Vanhoecke B, Iratni R, Takahashi T, Gaben AM, et al. Inhibition of cell survival, invasion, tumor growth and histone deacetylase activity by the dietary flavonoid luteolin in human epithelioid cancer cells. Eur J Pharmacol. 2011;651(1-3):18-25. https:// doi.org/10.1016/j.ejphar.2010.10.063. PMid:21074525.

86. Ng JM, Yu J. Promoter hypermethylation of tumour suppressor genes as potential biomarkers in colorectal cancer Int J MolSci. 2015;16(2):2472-2496. https://doi. org/10.3390/ijms16022472. PMid:25622259 PMCid:P MC4346847.

87. Abu-Serie MM, Habashy NH, Attia WE. In vitro evaluation of the synergistic antioxidant and antiinflammatory activities of the combined extracts from Malaysian Ganodermalucidum and Egyptian Chlorella vulgaris. BMC Complement Altern Med. 2018;18(1):154. https://doi.org/10.1186/s12906-0182218-5. PMid:29747629 PMCid:PMC5946467. 
88. Jayshree A, Jayashree S, Thangaraju N. Chlorella vulgaris and Chlamydomonasreinhardtii: effective antioxidant, antibacterial and anticancer mediators. Indian J Pharm Sci. 2016;78:575-581. https://doi. org/10.4172/pharmaceutical-sciences. 1000155.

89. Kunte M, Desai K. The protein extract of Chlorella minutissima inhibits the expression of MMP1, MMP-2 and MMP-9 in cancer cells through upregulation of TIMP-3 and down regulation of c-Jun. Cell J. 2018;20(2):211-219.

90. Meng TX, Irino N, Kondo R. Melanin biosynthesis inhibitory activity of a compound isolated from young green barley (Hordeumvulgare L.) in B16 melanoma cells. J Nat Med. 2015;69(3):427-431. https://doi. org/10.1007/s11418-015-0902-z. PMid:25827948.

91. Czerwonka A. Kawka K. Cykier K. Lemieszek MK, Rzeski W. Evaluation of anticancer activity of water and juice extracts of young Hordeumvulgare in human cancer cell lines HT-29 and A549. Ann Agric Environ Med. 2017;24(2):345-349. https://doi. org/10.26444/aaem/74714. PMid:28664721.

92. Fang YAO, Zhang JY, Xiang XIAO, Ying DONG, Zhou $\mathrm{XH}$. Antitumor activities and apoptosis-regulated mechanisms of fermented barley extract in the transplantation tumor model of human HT-29 cells in nude mice. Biomed Environ Sci. 2017;30(1):10-21.

93. Woo SM, Kwon SC, Ko SG, Cho SG. Barley grass extract causes apoptosis of cancer cells by increasing intracellular reactive oxygen species production. Biomed Rep. 2017;6(6):681-685. https://doi. org/10.3892/br.2017.897. PMid:28584641 PMCid:PM C5449973.

94. Vaško L, Vašková J, Fejerčáková A, Mojžišová G, Poráčová J. Comparison of some antioxidant properties of plant extracts from Origanumvulgare, Salvia officinalis, Eleutherococcussenticosus and Stevia rebaudiana. In Vitro Cell Dev BiolAnim. 2014;50(7):614-622. https://doi.org/10.1007/s11626014-9751-4. PMid:24737278.

95. Zhang XL, Guo YS, Wang CH, Li GQ, Xu JJ, Chung HY, et al. Phenolic compounds from Origanumvulgare and their antioxidant and antiviral activities. Food Chem. 2014;152:300-306. https://doi.org/10.1016/j. foodchem.2013.11.153. PMid:24444941.

96. Rubin B, Manso J, Monticelli H, Bertazza L, Redaelli M, Sensi F, et al. Crude extract of Origanumvulgare L. induced cell death and suppressed MAPK and PI3/ Akt signaling pathways in SW13 and H295R cell lines.
Nat Prod Res. 2019;33(11):1646-1649. https://doi.org /10.1080/14786419.2018.1425846. PMid:29334260.

97. Makrane H, El Messaoudi M, Melhaoui A, El Mzibri M, Benbacer L, Aziz M. Cytotoxicity of the aqueous extract and organic fractions from Origanummajorana on human breast cell line MDA-MB-231 and human colon cell line HT-29. Adv Pharmacol Sci. 2018; 2018:3297193. https://doi. org/10.1155/2018/3297193.PMid:30210537 PMCid:P MC6126111.

98. Al Dhaheri Y, Eid A, AbuQamar S, Attoub S, Khasawneh M, Aiche G, et al. Mitotic arrest and apoptosis in breast cancer cells induced by Origanum majorana extract: upregulation of TNF- $\alpha$ and down regulation of survivin and mutant p53. PLoS One. 2013;8(2):e56649. https://doi.org/10.1371/journal.po ne.0056649. PMid:23451065 PMCid:PMC3579842.

99. Al Dhaheri Y, Attoub S, Arafat K, Abuqamar S, Viallet J, Saleh A, et al. Anti-metastatic and anti-tumor growth effects of Origanummajorana on highly metastatic human breast cancer cells: inhi-bition of NFKB signaling and reduction of nitric oxide production. PLoS One. 2013;8(7):e68808. https://doi. org/10.1371/journal.pone.0068808. PMid:23874773 PMCid:PMC3707896.

100. Cortés-Rojas DF, de Souza CR, Oliveira WP. Clove (Syzygiumaromaticum): A precious spice. Asian Pac J Trop Biomed. 2014;4(2):90-96. https://doi. org/10.1016/S2221-1691(14)60215-X.

101. Dwivedi V, Shrivastava R, Hussain S, Ganguly C, Bharadwaj M. Comparative anticancer poten-tial of clove (Syzygiumaromaticum)--an Indian spice-against cancer cell lines of various anatomical origin. Asian Pac J Cancer Prev. 2011;12(8):1989-1993.

102. Aisha AF, Abu-Salah KM, Alrokayan SA, Siddiqui MJ, Ismail Z, Majid AM. Syzygiumaromaticum extracts as good source of betulinic acid and potential anti-breast cancer. Rev Bras Farmacogn. 2012;22(2):335-343.https://doi.org/10.1590/S0102$695 X 2011005000185$.

103. Pilátová M, Stupáková V, Varinská L, Sarisský M, Mirossay L, Mirossay A, et al. Effect of selected flavones on cancer and endothelial cells. Gen Physiol Biophys. 2010;29(2):134-43. https://doi.org/1 0.4149/gpb_2010_02_134. PMid:20577025.

104. Kim MY, Bo HH, Choi EO, Kwon DH, Kim HJ, Ahn $\mathrm{KI}$, et al. Induction of apoptosis by Citrus unshiu peel in human breast cancer MCF-7 cells: Involvement of 
ROS-dependent activation of AMPK. Biol Pharm Bull. 2018;41(5):713-721. https://doi.org/10.1248/bp b.b17-00898. PMid:29709909.

105. Kello M, Kulikova L, Vaskova J, Nagyova A, Mojzis J. Fruit peel polyphenolic extract-induced apoptosis in human breast cancer cells is associated with ROS production and modulation of p38MAPK/ Erk1/2 and the Akt signaling pathway. Nutr Cancer. 2017;69(6):920-931. https://doi.org/10.1080/016355 81.2017.1339819. PMid:28718669.

106. Shanmugapriya, Chen Y, Kanwar JR, Sasidharan S. Anticancer activity and molecular mechanism of polyphenol rich calophylluminophyllum fruit extract in MCF-7 breast cancer cells. Nutr Cancer. 2017;69(8):1308-1324. https://doi.org/10.1080/0163 5581.2017.1367944. PMid:29068745.

107. Hirsch K, Danilenko M, Giat J, Miron T, Rabinkov A, Wilchek M, et al. Effect of purified allicin, the major ingredient of freshly crushed garlic, on cancer cell proliferation. Nutr Cancer. 2000;38(2):245254. https://doi.org/10.1207/S15327914NC382_14 PMid:11525603.
108. El Khalki L, Tilaoui M, Jaafari A, Ait Mouse H, Zyad A. Studies on the dual cytotoxicity and anti-oxidant properties of Berberis vulgaris extracts and its main constituentberberine. Adv PharmacolSci.2018;2018: 3018498. https://doi.org/10.1155/2018/3018498. PM id:29805448 PMCid:PMC5817274.

109. Haba R, Watanabe S, Wada M, Udaka S. Effects of lactoferrin, soya germ and polyamine on 2-amino1-methyl-6-phenylimidazo [4,5-b]-pyridine(PhIP)induced breast carcinogenesis in rats. Biofactors. 2004;22(1-4):127-31. https://doi.org/10.1002/biof.55 20220125. PMid:15630267.

110. Oyenihi OR, Krygsman A, Verhoog N, de Beer D, Saayman MJ, Mouton TM, et al. Chemoprevention of LA7-induced mammary tumor growth by SM6Met, a well-characterized Cyclopia extract. Front Pharmacol. 2018;9:650. https://doi. org/10.3389/fphar.2018.00650. PMid:29973879 PMCid:PMC6019492.

111. Li J, Liu X, Chen H, Sun Z, Chen H, Wang L, et al. Multi-targeting chemoprevention of Chinese herb formula YangheHuayan decoction on experimentally induced mammary tumorigenesis. BMC Complement Altern Med. 2019;19(1):48. https://doi.org/10.1186/s12906-019-2456-1. PMid:30760265 PMCid:PMC6373088. 